\title{
LAS LAGUNAS DE RUIDERA DURANTE LA EDAD DEL BRONCE: UN TERRITORIO JERARQUIZADO
}

\author{
LAS LAGUNAS DE RUIDERA DURING BRONZE AGE: A HIERARCHICAL TERRITORY
}

\author{
ANDRÉS OCAÑA CARRETÓN $(*)$
}

\begin{abstract}
RESUMEN
Este artículo muestra los primeros resultados de un proyecto que tiene por objeto analizar la relación espacial entre los recursos y los asentamientos en el Alto Guadiana como aproximación a los sistemas productivos de las comunidafes que habitaron este territorio durante el Calcolítico y la Edad del Bronce. En concreto, se analiza el patrón de asentamiento durante la Edad del Bronce en el Parque Natural de las Lagunas de Ruidera, el cual indica la existencia de una ocupación jerárquica del territorio como reflejo de la existencia de un cierto grado de complejidad social.
\end{abstract}

\begin{abstract}
This paper shows the first results of a research project that aims to analyse the spatial relationship betwen the natural resources and the settlements in the Upper Guadiana River (South Meseta, Spain). The data obtained will be used to understand the productive system of the communities which lived in this territory during the Copper and Bronze Ages. In particular the settlement pattern during the Bronze Age is analysed in the Nature Reserve of Lagunas de Ruidera. This shows a territorial hierarchical structure and allows us to understand the emergence of social complexity within this territory.
\end{abstract}

Palabras clave: Lagunas de Ruidera. Edad del Bronce. Patrones de asentamiento. Jerarquización territorial. Complejidad social.

Key words: Lagunas de Ruidera. Bronze Age. Settlement patterns. Territorial hierarchical structure. Social complexity.

(*) Académicos, 3. 13710 Argamasilla de Alba. Ciudad Real. Correo electrónico: andresocana@ navegalia.com Recibido: 29-I-2001; aceptado: 26-VI-2001

\section{INTRODUCCIÓN}

Este trabajo presenta los primeros resultados de un proyecto de investigación que tiene por objeto el estudio de los patrones de asentamiento durante el III y II milenios a.C. en el Alto Guadiana, siendo la finalidad última analizar el proceso que lleva a la aparición de la complejidad social durante las primeras etapas metalúrgicas en el territorio elegido y cuales son los elementos que lo caracterizarían. En concreto, se analiza la información obtenida tras el desarrollo de la primera fase del proyecto, consistente en la localización y estudio de los yacimientos conocidos por la bibliografía (Nájera y Molina, 1977; Jiménez y Chaparro, 1989; López y Fernández, 1994; Rico et al., 1994; Fernández-Miranda et al., 1994; Colmenarejo et al., 1998). Ésta nos ha permitido realizar una aproximación al patrón de asentamiento existente en las Lagunas de Ruidera durante la Edad del Bronce, el cual parece mostrar indicios de una incipiente ordenación jerárquica.

\section{EL TERRITORIO: LAS LAGUNAS DE RUIDERA}

Las Lagunas de Ruidera constituyen un singular fenómeno que divide al Alto Guadiana en dos tramos (ver Fig. 1). Éstas forman un conjunto de 15 lagunas a lo largo de $25 \mathrm{~km}$. que se disponen de manera escalonada, cerrada cada una de ellas por una barrera travertínica a modo de represa natural (González et al., 1987: 228). Desde el punto de vista geológico se localizan dentro del Campo de Montiel, aunque ya en su zona de contacto con la llanura manchega en la que se ubican algunos de los yacimientos aquí estudiados. El Campo de Montiel constituye una plataforma morfoestructural cuyo 


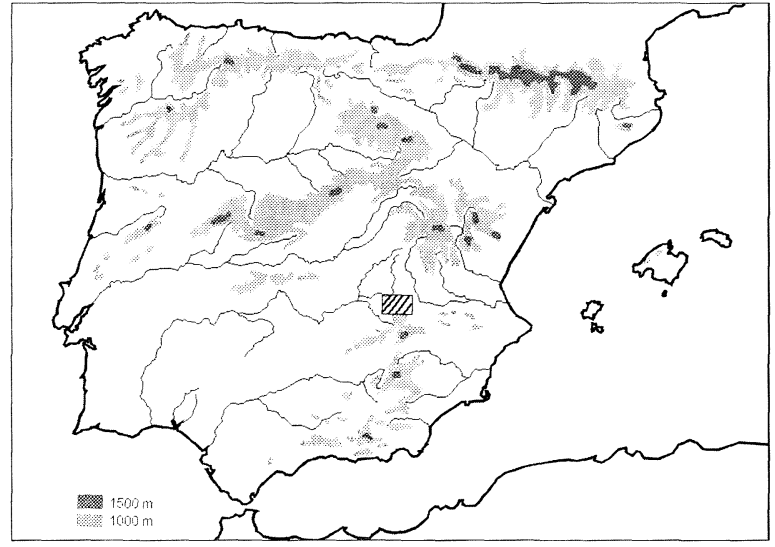

Fig. 1. Localización de la zona estudiada.

relieve es el resultado de diversas plataformas de erosión miocénicas de las que la más reciente es la superficie de Ossa de Montiel (1). Ésta constituye una planicie espectacular, trabajada en los tramos inferior y medio del Lías en la que el Alto Guadiana y su red tributaria ha modelado un relieve de valles estrechos y torrenteras, entre las que se extienden zonas amesetadas -prácticamente llanas o con suaves ondulaciones- que reciben la denominación de mesas. En este paisaje los cerros aislados apenas existen y los mayores desniveles se sitúan en los bordes de los valles, especialmente del principal, donde las pendientes superan fácilmente el $20 \%$. En la zona correspondiente a la llanura manchega, la morfología es prácticamente llana, y tan sólo se ve alterada por suaves ondulaciones paralelas al discurrir del río que llegan hasta el paraje conocido como Santa María. Se trata de depósitos de cantos con escasa matriz, relacionados con el abanico aluvial del Alto Guadiana (2). A partir de este punto el relieve es totalmente llano.

En las Lagunas de Ruidera y su entorno predominan los suelos pardo-rojizos calizos, las xerorendsinas y los litosuelos (Peinado y Martínez, 1985: 141). Agronómicamente hablando, se trata de suelos de tipo medio, con abundante piedra suelta que obliga a frecuentes labores de despedregado para su cultivo. Su aprovechamiento, básicamente, es para secano, siendo óptimos también para cultivos arbustivos del tipo vid.

(1) Pérez, A. (1982): Neógeno y Cuaternario de la llanura manchega. Universidad Complutense de Madrid (Colección Tesis Doctorales), Madrid, págs. 281-284.

(2) Pérez (1982): 389, nota 1

\section{LOS ASENTAMIENTOS}

La zona estudiada comprende unos $52 \mathrm{~km}$ lineales, de los que 44 corresponden al valle del Alto Guadiana en el tramo comprendido entre la laguna Blanca y la población de Argamasilla de Alba y el resto a un tramo del arroyo Alarconcillo y a otro de la Cañada de las Hazadillas -ambos afluentes del primero-. Aquí hemos localizado y estudiado un total de 29 yacimientos, de los que 21 se encuentran en el valle principal, 6 en el Arroyo Alarconcillo y 2 en la Cañada de las Hazadillas. Estos números nos están indicando la existencia de una importante concentración de yacimientos (3), que para los casos más acusados como el del Arroyo Alarconcillo podría, en un principio, ser atribuido a lo reducido del tramo estudiado -4,5 km-. No obstante, el análisis de las distancias al vecino más próximo (4) parece corroborar esa primera impresión, además de apuntar una cierta tendencia hacia un patrón agrupado.

De los 29, 6 se sitúan en el fondo del valle, buscando siempre ambientes de tipo palustre, y carecen de defensas naturales, si exceptuamos el caso del castillo de Rochafrida, localizado en el fondo del valle sobre un pequeño cerrete formado por un afloramiento de calizas. Los otros 23 se localizan en lugares elevados, generalmente de fácil defensa, con pendientes que superan el $20 \%$ y una altura relativa media de $47 \mathrm{~m}$. Los lugares elegidos son, preferentemente, espolones o salientes del relieve hacia el valle con muy buenas defensas naturales en todos sus lados, salvo en la zona de contacto con el relieve circundante. Una constante documentada es la predilección por su ubicación en lugares próximos al fondo del valle, buscando un buen control visual sobre el mismo, estando la distancia media de los yacimientos al valle en el que se sitúan en $142 \mathrm{~m}$ (5). No obstante, hemos documentado algunas desviaciones significativas a este patrón, de las que el caso más llamativo sería Casa del Gavilán -1.250 m-y cuyas posibles causas serán analizadas más adelante. Por último, indicar que creemos que la elección del lugar para la localización de los asentamientos estaría motivada principalmente por su funcionalidad, si bien cabe mencionar que existe un factor de

(3) 1 yacimiento cada $2 \mathrm{~km}$ en el valle del Alto Guadiana; 1 yacimiento cada $0,75 \mathrm{~km}$. en el valle del Arroyo Alarconcillo; y 1 yacimiento cada $1,75 \mathrm{~km}$ en la Cañada de las Hazadillas.

(4) $1.175 \mathrm{~m}$ de media en el valle del Alto Guadiana y $600 \mathrm{~m}$ en el valle del Arroyo Alarconcillo.

(5) $168 \mathrm{~m}$ en el valle del Alto Guadiana y $71 \mathrm{~m}$ en el del Arroyo Alarconcillo.

T. P., 59, n. ${ }^{\circ} 1,2002$ 


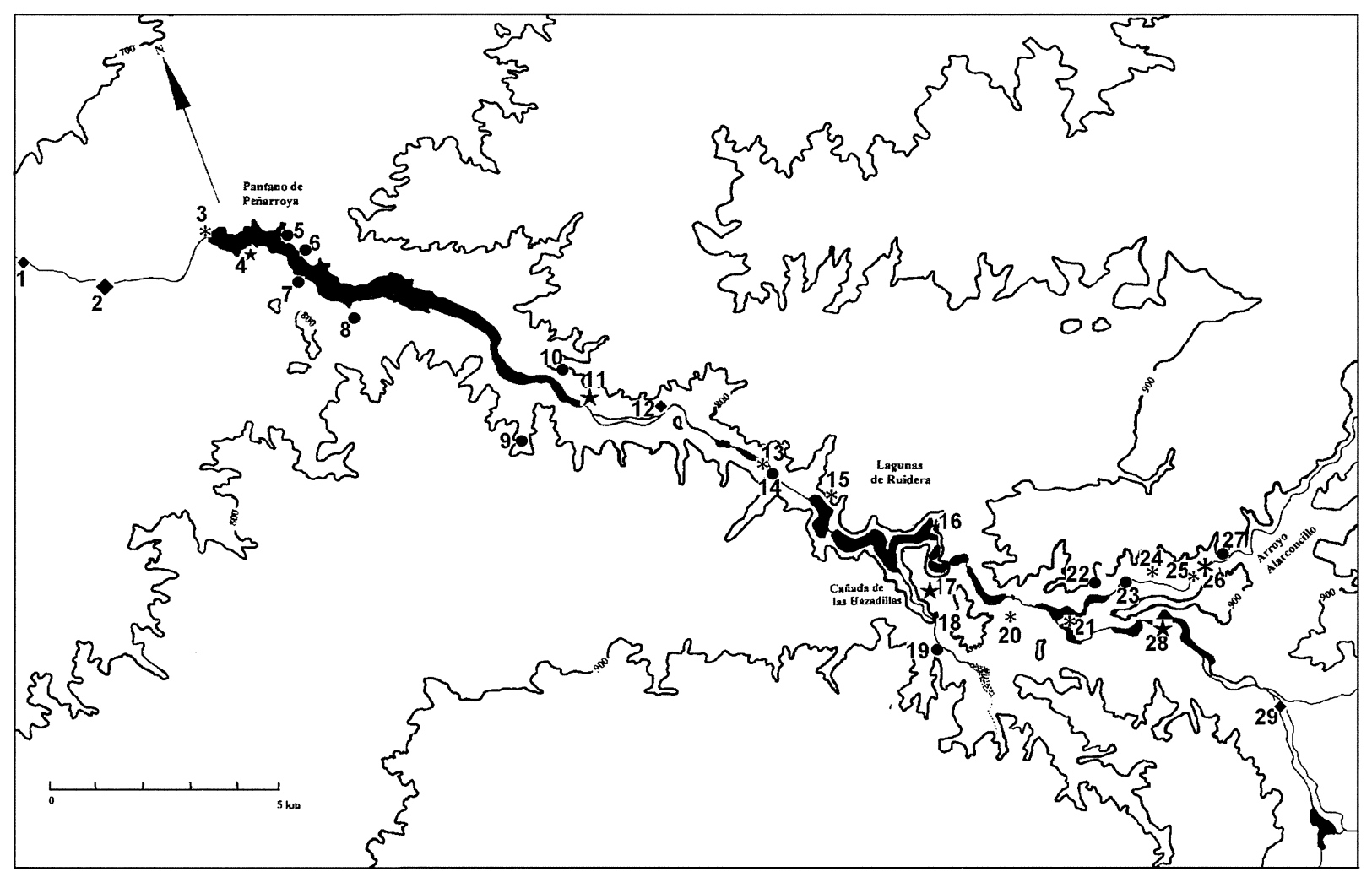

Fig. 2. Yacimientos localizados en las Lagunas de Ruidera, diferenciados según modelos de poblamiento y tamaño. La numeración se corresponde con la aparecida en la tabla 2. Poblados fortificados de planta circular localizados en llano inferiores a $2.000 \mathrm{~m}^{2}(\diamond)$, superiores a $2.000 \mathrm{~m}^{2}(\diamond)$; poblados fortificados de planta circular localizados en alto $(\bullet)$; poblados fortificados de planta circular localizados en alto con espacios delimitados con muros inferiores a $2.000 \mathrm{~m}^{2}(\star)$, superiores a $2.000 \mathrm{~m}^{2}(\star)$; poblados sin patrón arquitectónico homogéneo inferiores a $2.000 \mathrm{~m}^{2}(*)$, superiores a $2.000 \mathrm{~m}^{2}(*)$

limitación que vendría impuesto por el relieve, ya que hay partes del valle en las que sólo es posible el asentamiento en lugares elevados. A su vez, también llama la atención el hecho de que siempre que las características del valle lo permiten -ensanchamiento de éste- se producen localizaciones en su fondo, buscando ambientes palustres, como sucede en los casos de Moraleja-2, Jacidra, Cueva Moreni1la-1 y las motillas de Retamar y Santa María.

La totalidad de los yacimientos localizados presentan, en general, un tamaño reducido y relativamente homogéneo, estando el $77 \%$ de la muestra entre los $200 \mathrm{y} \operatorname{los} 1.500 \mathrm{~m}^{2}$. La distribu- ción de éstos según tamaños en $\mathrm{m}^{2}$ se encuentra en la tabla 1.

Como se puede observar en esta tabla existen tres yacimientos que superan los $3.000 \mathrm{~m}^{2}$, saliéndose de una manera significativa de la media del conjunto. Se trata del Castillo de Rochafrida, de Cerro Chicano y de la Mesa del Almendral, éste último con una extensión por encima de la hectárea.

En relación al tamaño cabe destacar dos aspectos interesantes. Por un lado, estaría el hecho de que los yacimientos de mayores dimensiones mantengan una cierta regularidad en las distancias que los separan. Así Mesa del Almendral está separado tanto del

\begin{tabular}{|c|c|c|c|c|c|}
\hline $\mathbf{0 - 5 0 0}$ & $\mathbf{5 0 0 - 1 5 0 0}$ & $\mathbf{1 5 0 0 - 3 0 0 0}$ & $\mathbf{3 0 0 0 - 5 0 0 0}$ & $\mathbf{5 0 0 0 - 1 0 0 0 0}$ & $\mathbf{+}$ de10000 \\
\hline 13 & 8 & 3 & 1 & 1 & 1 \\
\hline
\end{tabular}

Tab. 1. Tamaño en $\mathrm{m}^{2}$ de los yacimientos. 


\begin{tabular}{|c|c|c|c|}
\hline $\mathrm{N}^{0}$ & Nombre & Ext. $\mathrm{m}^{2}$ & Caracteristicas \\
\hline 1 & Motilla de Santa María & 1.600 & Motilla \\
\hline 2 & Motilla del Retamar & 3.000 & Motilla con ocup. ibérica \\
\hline 3 & Castillo de Peñarroya & Indeterm. & Poblado indeter. bajo fortificación medieval \\
\hline 4 & Mesas de la Parra-1 & 1.252 & Morra con poblado amurallado \\
\hline 5 & Altarejos-1 & 1.171 & Morra \\
\hline 6 & Altarejos-2 & 870 & Morra \\
\hline 7 & Poblado de Despeñaperros & 1.206 & Morra \\
\hline 8 & Huerta de Aguas-2 & 1.100 & Morra \\
\hline 9 & Casa del Gavilán & 400 & Morra \\
\hline 10 & Grederas & 252 & Morra \\
\hline 11 & Cerro Chicano & 5.854 & Morra con poblado amurallado \\
\hline 12 & Moraleja-2 & 1.000 & Motilla parcialmente desmantelada \\
\hline 13 & Cueva Morenilla-1 & 100 & Indeterminado \\
\hline 14 & El Castillón-1 & 516 & Morra \\
\hline 15 & Laguna Rey-1 & 25 & Indeterminado \\
\hline 16 & Laguna Colgada-1 & 620 & Poblado sin estructuras \\
\hline 17 & Mesa del Almendral & +10.000 & $\begin{array}{l}\text { Morra con poblado amurallado y ocup. Bronce } \\
\text { Final/Hierro I }\end{array}$ \\
\hline 18 & Hazadillas-12 & 460 & Morra \\
\hline 19 & Hazadillas-8 & 400 & Morra \\
\hline 20 & Salto del Fraile & 600 & Poblado con estructuras \\
\hline 21 & Cerro de los Almorchones & Indeterm. & $\begin{array}{l}\text { Poblado indeterminado con ocup. ibérica, } \\
\text { romana y medieval }\end{array}$ \\
\hline 22 & Quebrada del Toro-1 & 416 & Morra \\
\hline 23 & San Pedro-1 & 201 & Morra \\
\hline 24 & Cuesta Almagra & 200 & Poblado sin estructuras \\
\hline 25 & Arroyo Alarconcillo-1 & 200 & Poblado indeterminado \\
\hline 26 & Castillo de Rochafrida & 3.780 & Poblado indeter bajo fortificación medieval \\
\hline 27 & El Tobar-1 & 390 & Morra \\
\hline 28 & Era Vieja-1 & 2.200 & Morra con poblado amurallado \\
\hline 29 & La Jacidra-1 & Indeterm. & Motilla desmantelada \\
\hline
\end{tabular}

Tab 2. Relación de yacimientos estudiados.

Castillo de Rochafrida como de Era Vieja-1 unos 5,5 $\mathrm{km}$.; de Cerro Chicano estaría a $8 \mathrm{~km}$.; distancia similar a la que separa éste último de Mesas de la $\mathrm{Pa}$ rra-1 (6). A su vez se constata la existencia de una cierta relación de proporcionalidad inversa entre los yacimientos de mayor tamaño y los localizados en su entorno. Así los yacimientos de mayor tamaño como Cerro Chicano, Mesas del Almendral y castillo de Rochafrida presentan en su entorno yacimientos que rara vez superan $\operatorname{los} 500 \mathrm{~m}^{2}$, mientras que en el caso de Mesas de la Parra-1 $\left(1.250 \mathrm{~m}^{2}\right)$ los yacimientos de su entorno presentan un tamaño homogéneo y similar al suyo, que rara vez baja de los $1.000 \mathrm{~m}^{2}$. Por otro, estaría el hecho de que los yaci-

(6) Éste no es un yacimiento de los de mayor tamaño, pero sí presenta una diferenciación en cuanto a su estructura con los de su entorno. Es un poblado fortificado de planta central localizado en altura que presenta un espacios delimitado por una línea de muro, mientras que los de su entorno sólo presentan el núcleo fortificado. mientos de mayor tamaño son, por lo general, los que mayor número de contactos visuales presentan. Estos suelen mantener contacto con dos o mas yacimientos, mientras que lo normal es que el resto lo mantenga con uno, o ninguno. Éste constituye un aspecto importante dentro del patrón, que queda reflejado en el registro arqueológico en la elección del lugar para el emplazamiento de algunos asentamientos, caso de Altarejos-2. Éste es el único poblado de altura fortificado de planta circular que carece de defensas naturales. La razón de ello hay que buscarla en la necesidad de ubicarse en un lugar que permitiera el contacto visual con Mesas de la Parra1 y el Poblado de Despeñaperros, ya que presenta zonas próximas con buenas defensas naturales, pero en las que dicho contacto no es posible.

\subsection{Modelos de asentamiento}

El patrón observado se caracteriza por la existencia de una cierta variedad entre los modelos de asentamiento documentados, si bien éstos pueden ser agrupados básicamente en tres, bien representados en zonas próximas como la Mancha Oriental (Fernández-Miranda et al., 1994) y la Mancha Toledana (Ruiz Taboada, 1998), y que serían:

- Poblados fortificados de planta circular localizados en llano.

- Poblados fortificados de planta circular localizados en altura.

- Poblados sin un patrón arquitectónico homogéneo.

Ahora bien, en el caso del territorio estudiado, tenemos noticias, aún no suficientemente contrastadas, de la posible existencia de otros modelos, como sería el caso de la ocupación de cuevas. Las características geológicas de la zona permiten la formación de pequeñas cavidades o abrigos, en algunos de los cuales se han documentado cerámicas a mano que podrían ser atribuidas a la Edad del Bronce (7). No obstante, dado el escaso conocimiento que aún tenemos del período precedente resulta complicado precisar si estos materiales corresponden a este momento o a momentos anteriores, o tal vez a ambos, así como la funcionalidad de las ocupaciones -funeraria o hábitat-. A este res-

(7) Se trata de materiales depositados en el Museo de Albacete procedentes de la cueva de Montesinos y de unos pequeños abrigos en la margen izquierda de la laguna Lengua. Aprovechamos la ocasión para mostrar nuestro agradecimiento a su directora Dña. Rubí Sanz Gamo por su amabilidad y facilidades dadas para su consulta. 
pecto creemos que los trabajos que venimos desarrollando en Cueva Maturras (Gutiérrez et al., 2000) serán de gran utilidad para arrojar algo de luz sobre la posible funcionalidad y cronología de estas ocupaciones, que parecen corresponder, más bien, a las etapas precedentes y en general tendrían un carácter funerario.

\subsubsection{Poblados fortificados de planta circular localizados en llano}

Popularmente son conocidos con el nombre de motillas. En total hemos localizado cuatro que, a excepción de la motilla del Retamar, presentan un importante grado de deterioro que, en algunos casos, ha llevado a su práctica total desaparición (8).

La motilla del Retamar (9) es el yacimiento mejor conocido de este grupo y de la zona al haber sido objeto de varias campañas de excavación (10). Presenta la estructura típica de este modelo formada por dos anillos de muralla dispuestos de manera seudoconcéntrica, en cuyo centro se incluye una posible torre (Galán y Sánchez, 1994). Los espacios entre dichos anillos son aprovechados para la ubicación de las viviendas, no habiéndose documentado restos de éstas al exterior de los anillos, a diferencia de lo documentado en otros yacimientos de este grupo como las motillas del Azuer y Los Palacios. Ésta, creemos que debió de ser una constante entre los asentamientos pertenecientes a este modelo documentados en la zona debido al carácter palustre de los lugares elegidos para su ubicación, lo que haría prácticamente imposible la ubicación de viviendas al exterior del último recinto amurallado que, además de una función defensiva, es muy probable que también cumpliera una función de dique ante posibles subidas del nivel hídrico.

(8) Éste sería el caso de Jacidra, totalmente desmantelada y de la que ya sólo quedan algunos indicios. Moraleja-2 también se encuentra parcialmente desmantelada. Fuera de la zona estudiada, pero en sus proximidades tenemos los casos de las motillas de la Membrilleja (Nájera y Molina, 1977), Dos Barrios y Perales, las tres totalmente desmanteladas.

(9) Denominada por sus excavadores motilla de Santa María del Retamar. Nosotros hemos optado por la denominación tradicional de motilla del Retamar, conocida así por estar junto a la casa del mismo nombre, al considerar que la otra se presta a confusión al utilizar para su denominación el nombre unido de las dos motillas existentes en esta zona, Santa María y Retamar.

(10) Para una mayor información remitimos a las diversas publicaciones que existen sobre la misma incluidas en la bibliografía final.

\subsubsection{Poblados fortificados de planta circular localizados en altura}

Constituyen el modelo mayoritario en la zona con un total de 16 yacimientos. Tradicionalmente son conocidos con el nombre de morras, aunque también pueden presentar otras denominaciones, como sucede en el caso de El Castillón-1. Se localizan, generalmente, sobre espolones o salientes del relieve con buenas defensas naturales en todos sus lados, salvo en el que conecta con el relieve circundante, zona elegida para la localización de la estructura fortificada de planta circular que caracteriza el modelo. En algunos casos, a ésta se asocian pequeñas dispersiones de material, generalmente lítico, que podrían estar indicando la existencia de zonas de hábitat asociadas a las fortificaciones, cuya extensión resulta difícil de precisar, pero que en ningún caso debió ser excesivamente grande. En general, presentan un tamaño reducido, oscilando entre los 200 y los $1.200 \mathrm{~m}^{2}$, con una media de $620 \mathrm{~m}^{2}$, si bien tres de ellos -Cerro Chicano, Mesa del Almendral y Era Vieja-1-no han sido incluidos en ésta al desviarse de manera significativa de la misma. Para este modelo no contamos con información procedente de excavaciones, si bien los trabajos realizados por un grupo de Misión Rescate en el poblado de Despeñaperros nos permiten una aproximación a sus características. Dicha actuación puso al descubierto una estructura circular de unos $7 \mathrm{~m}$ de diámetro que parece corresponder a una torre, en torno a la cual se observan muros de tendencia circular en superficie. Disposiciones superficiales similares se observan en otros asentamientos como Altarejos1, Huerta de Aguas-2 Altarejos-2, Mesas de la Parra-1, Quebrada del Toro-1, Era Vieja-1, el Tobar1 y Mesa del Almendral. Estas características permiten suponer que este modelo responde a una concepción arquitectónica muy similar a la de los localizados en llano, radicando su única diferencia en el lugar elegido para su ubicación. Sin embargo en algunos casos, como Hazadillas-8 y Cerro Chicano, la estructura se simplifica, reduciéndose a un único muro de tendencia circular. Esta simplificación, no obstante, también ha podido ser recientemente documentada en algunos yacimientos fortificados de llanura localizados al norte de la población de Argamasilla de Alba (11), lo que vendría a corroborar

(11) Se trata de la motilla de Juez, pequeño asentamiento de llanura compuesto por un único muro de tendencia circular, ubicado en la margen derecha del Alto Guadiana. Similar estructura tendría la motilla de Pedregoso, ya en la margen izquierda del río Záncara. 
la similitud del patrón arquitectónico entre ambos modelos. Por último, cabe mencionar la existencia de un reducido grupo dentro de éstos, caracterizado por la presencia de líneas de muro que partiendo de la estructura fortificada delimitarían uno, o más espacios. Se trata de Mesas de la Parra-1, Era Vieja-1, Cerro Chicano y Mesa del Almendral, estando, a excepción de Mesas de la Parra-1, entre los de mayor extensión del total de los analizados. En los cuatro casos, la fortificación se localiza en la zona más accesible -zona de contacto con el relieve circundante-, extendiéndose el poblado hacia el final del relieve y completándose las defensas naturales con defensas artificiales.

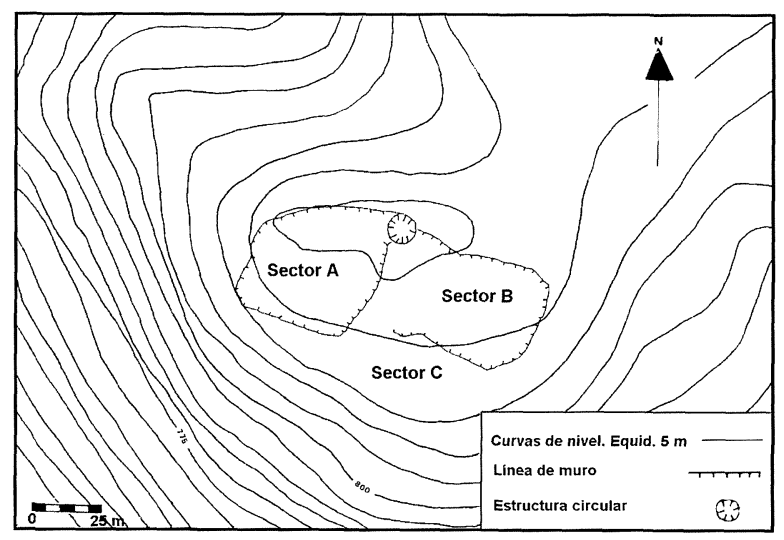

Fig. 3. Cerro Chicano.

Cerro Chicano es el que mayor complejidad presenta. Posee una estructura circular -ver figura 3 - de la que parten varias líneas de muro que forman dos recintos, que a su vez sirven para delimitar un tercer espacio entre éstos y el final del relieve. Mesa del Almendral es el yacimiento que presenta una mayor extensión, aunque su estimación total presenta algunos problemas derivados de la existencia de otra ocupación posterior, atribuible al Bronce Final/Hierro I (Ocaña y Gómez, 2000). La superficie total del yacimiento es de unas 12 has., que nosotros hemos dividido en dos sectores -ver figura 4-, localizándose las estructuras atribuibles a la Edad del Bronce en el Sector B, que presenta una extensión aproximada de 5 has. Estas están compuestas por una estructura fortificada de planta circular de reducidas dimensiones $\left(450 \mathrm{~m}^{2}\right)$ que cierra el acceso al yacimiento por el sur de la que parten dos líneas de muro, una que va hacia el oeste y otra que va hacia el noreste. El hecho de que el espacio no esté delimitado de manera clara en el

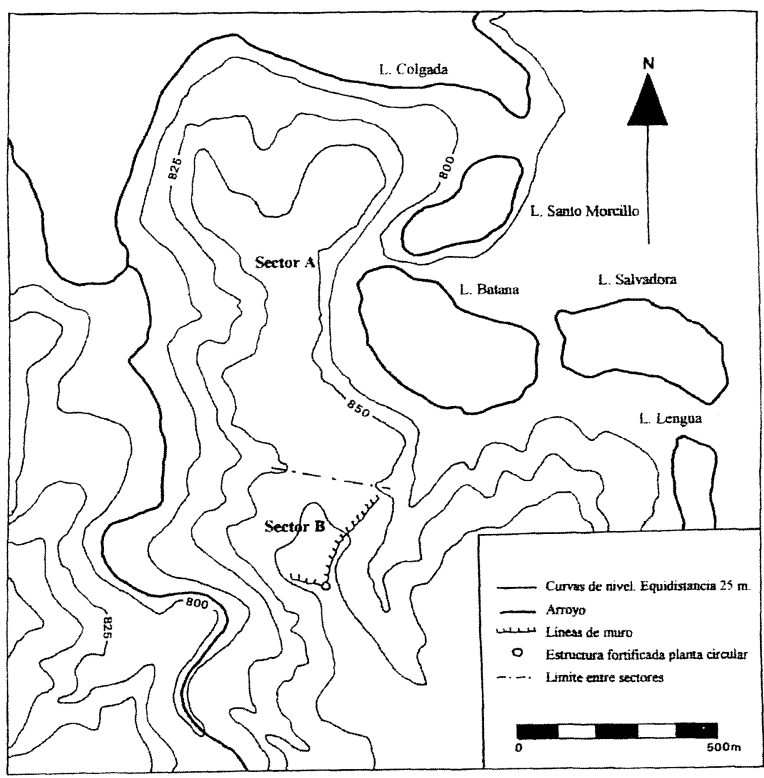

Fig. 4. Mesa del Almendral.

lado norte nos hace que tomemos con ciertas reservas la cifra de 5 has. que ocuparía el Sector B como extensión, si bien creemos que es fácil suponer que supere la hectárea. Los dos asentamientos restantes están formados por la estructura fortificada y una línea de muro que delimita un único espacio.

\subsubsection{Poblados sin un patrón arquitectónico homogéneo}

En este modelo hemos incluido 9 yacimientos, que se caracterizan por presentar una cierta variedad en cuanto a sus localizaciones, características y tamaño. Unos se sitúan en relieves aislados, por otra parte muy escasos en la zona, presentando una variada localización que va del fondo del valle castillo de Rochafrida- a zonas elevadas -Salto del Fraile-, pasando por suaves relieves entre dos lagunas -cerro de los Almorchones-. Otros se presentan en salientes del relieve-Arroyo Alarconcillo-1-, o a media ladera -Laguna del Rey-1-. Un caso peculiar es el de Cueva Morenilla-1, localizado en un emplazamiento similar al elegido para los poblados fortificados de planta circular localizados en llano -fondo de valle en un ambiente palustre-. Éste no presenta ningún tipo de estructura observable en superficie, aunque visto de lejos presenta el mismo aspecto que aquellos. El asentamiento aprovecha un pequeño afloramiento de travertino junto a la 
laguna Cueva Morenilla, despreciando otras potenciales localizaciones (12).

En cuanto a la presencia de fortificaciones, cabe indicar que tan sólo ha podido ser documentada con seguridad en el caso de Salto del Fraile, si bien también creemos muy probable que estuvieran presentes en los castillos de Rochafrida y Peñarroya. Otros creemos que carecerían de ellas, como sería el caso de Arroyo Alarconcillo-1 y Laguna del Rey-1. Por último indicar que su tamaño oscila entre los $25 \mathrm{~m}^{2}$ escasos que presenta Laguna del Rey-1 y los 3.780 $\mathrm{m}^{2}$ que presenta el castillo de Rochafrida, no habiendo sido posible estimar el tamaño en algunos casos como el del castillo de Peñarroya y cerro de los Almorchones.

\subsection{Territorios de explotación}

El análisis de los territorios de explotación pone de manifiesto la existencia de tres grupos dentro de los asentamientos estudiados. Uno, y más numeroso, en el que el uso mayoritario es el monte, otro en el que el uso mayoritario es el secano y un tercero en el que monte y secano se presentan equilibrados -sólo representado por Huerta de Aguas-2 y Casa del Gavilán-. A su vez, también pone de manifiesto, a pesar de ser una zona con importantes recursos hídricos, la escasa representatividad del regadío, salvo en muy contados casos, que se reducen a los yacimientos localizados en los fondos de valle. Estos aspectos nos hacen pensar en la existencia de una posible diferenciación funcional entre los asentamientos, que se hace más evidente en casos como Huerta de Aguas-2 y Casa del Gavilán, ambos enclavados en zonas con un buen potencial agrícola en su entorno más inmediato, pero alejados del patrón habitual de control visual sobre los fondos de valle. Ésta, a su vez, también podría ser la explicación para la ocupación de los fondos de los valles siempre que las características orográficas lo permiten, por ser éstos lugares más aptos para un aprovechamiento intensivo, tanto agrícola como ganadero, siendo éste el caso de Moraleja-2, Jacidra, castillo de Rochadrida, motillas del Retamar y Santa María y Cueva Morenilla-1.

El análisis de los territorios de explotación, por otra parte, también nos ha permitido comprobar la

(12) Próximo al elegido existen restos de otra pequeña terraza de travertino, fuera del fondo del valle y en un lugar ligeramente más elevado, que sin embargo no ha sido seleccionado, prefiriendo la localización en el fondo del valle. ausencia de proporcionalidad entre el tamaño de los asentamientos y el de sus territorios de explotación. Esta desproporción se hace más evidente en el caso de los asentamientos de mayor tamaño como Cerro Chicano y Mesa del Almendral cuyos territorios de explotación son sensiblemente más reducidos que los de los yacimientos más próximos, los cuales presentan un tamaño sensiblemente inferior. Esta circunstancia creeemos que puede ser interpretada en dos direciones. Por una parte, podría ser el reflejo de la existencia de apropiación de excedentes de los asentamientos de mayor tamaño respecto a los de menor tamaño, y por otra podría estar indicando la existencia de una intensificación económica entre los asentamientos de mayor tamaño, lo que les permitiría sustentar a una mayor población con un territorio menor. No obstante consideramos que ambas no son incompatibles y podrían ser tomados como indicios de la existencia de una jerarquización territorial.

\subsection{Los materiales}

La información que poseemos sobre los materiales es muy limitada debido a las características de la recogida (13), si bien en algunos aspectos se ve complementada por la proporcionada por los trabajos de excavación en la motilla del Retamar. El material más abundante es la cerámica que se caracteriza por presentar, en general, buenos acabados -alisado y bruñidos-, y ser en su gran mayoría lisa, limitándose la decoración a impresiones de punzón, digitaciones, ungulaciones, o a una combinación de estas dos últimas sobre los borde de las vasijas. También hemos documentados mamelones, generalmente colocados bajo el labio, o sobre la línea de carena, que en muchos casos presentan un carácter decorativo. Llama la atención, por su carácter excepcional, la presencia de dos fragmentos superficiales con decoración de tipo Dornajos, uno procedente de la motilla de Santa María (Nájera y Molina, 1977: 256), y el otro sin procedencia exacta (14). Por último indicar que entre las formas destacan los cuencos, las ollas, los vasos carenados de variada morfología y los recipientes de gran tamaño. En general, se trataría de producciones locales,

(13) Se realizó una recogida selectiva, limitándose a aquellos fragmentos más interesantes para un diagnóstico crono-cultural.

(14) Se trata de un fragmento procedente de la zona de la Moraleja, dado a conocer por un aficionado local (Jiménez y Chaparro, 1989), aunque sin precisar su origen. 
como así parece confirmarlo un estudio geoquímico y mineralógico (Capel, 1987) realizado sobre una muestra de cerámicas procedentes de yacimientos manchegos de la Edad del Bronce, entre los que se encuentran tres de la zona estudiada (15), si bien resulta de interés el hecho de que uno de los fragmentos procedentes del poblado de Despeñaperros tenga un carácter alóctono. Esta circunstancia vendría a probar la existencia de intercambios con comunidades de otros territorios, ya que ésta sería la única explicación posible para su presencia en este yacimiento.

La industria lítica es escasa y de poco interés. Las materias primas utilizadas son la cuarcita y el sílex. La primera es abundante en la zona, si bien hemos documentado una peculiaridad en los asentamientos de la zona de las lagunas -no así en el tramo del embalse-, que es su doble origen. Una parte procede de cantos de río -abundantes en la zona-, mientras que la otra del único afloramiento del zócalo primario existente en la zona y que está compuesto por cuarcitas -Cuesta de la Almagra-, sitio elegido para la localización de un asentamiento que es muy probable que estuviera relacionado con la explotación y extracción de esta materia. El sílex no presenta sitios de aprovisionamiento en la zona (16), siendo la materia prima elegida en la mayoría de las piezas que hemos podido clasificar tipológicamente, y que se limitan a fragmentos de lámina y denticulados, algunos de éstos últimos con lustre de cereal. Los pulimentados sólo se encuentran presentes en la motilla del Retamar, si bien destaca la presencia en Cerro Chicano de una pieza cuya morfología recuerda la de un hacha pulimentada, pero que presenta la peculiaridad de estar realizada en arenisca.

La presencia de otros materiales tan sólo la conocemos por las excavaciones en la motilla del Retamar (Colmenarejo et al., 1988; Hernando y Galán, 1989; Galán y Sánchez, 1994). Entre éstos se encuentra el metal del que se han recuperado 12 puntas de flecha, 3 puñales de remaches y diversos punzones de cobre. El lugar más próximo para el aprovisionamiento de mineral (17) se encuentra en la zona de Madridejos y Camuñas (Montero Ruiz

(15) Motilla de Santa María, Motilla del Retamar y Poblado de Despeñaperros.

(16) Los lugares más próximos se encuentran en Campo de Criptana y Villarrobledo a unos $60 \mathrm{~km}$ en ambos casos.

(17) Ya comentamos que, en la hoja 788 del Mapa Geológico de España 1:50.000, se habla de la presencia de azurita y malaquita en Cuesta de la Almagra, si bien desconocemos sus posibilidades de explotación. et al., 1990), cuya distancia a la zona es de unos 100 $\mathrm{km}$, aproximadamente. Éste se transformaría en el yacimiento como así parecen indicarlo la presencia de escorias y de una posible tobera de horno (Colmenarejo et al., 1988: 88). Por último, destacar también la existencia en este yacimiento de marfil botón de perforación en $\mathrm{V}$ - y conchas perforadas de moluscos, entre las que cabe mencionar la presencia de algunas de origen marino.

\section{A MODO DE CONCLUSIÓN: LAS LAGUNAS DE RUIDERA UN TERRITORIO JERARQUIZADO}

El patrón de asentamiento documentado en las Lagunas de Ruidera durante la Edad del Bronce parece apuntar la existencia de una incipiente jerarquización territorial. Así parecen indicarlo las diferencias documentadas en el tamaño de los asentamientos y el importante grado de fortificación que presenta la mayoría de éstos; la tendencia a un patrón agrupado y al control visual sobre el fondo de los valles; la aparente diferenciación funcional respecto a los recursos explotables; así como la posible apropiación de excedentes e intensificación económica.

La presencia de una diferenciación en el tamaño de los asentamientos suele ser uno de los elementos más utilizados a la hora de establecer la existencia de jerarquización territorial. No obstante, su aceptación suele requerir la superación de una serie de limitaciones, entre las que cabe citar, la certeza de poseer una muestra representativa de los asentamientos, la contemporaneidad de éstos y la posibilidad de establecer de manera fiable su extensión. En el caso que nos ocupa, al igual que se ha sugerido para otros territorios próximos (Fernández-Miranda et al., 1994: 265), creemos que éstas pueden ser minimizadas. Por una parte, las características de los asentamientos documentados y la ausencia de una actividad agrícola importante en la zona es muy probable que nos hayan permitido documentar la práctica totalidad de los asentamientos atribuibles a la Edad del Bronce (18). Por otra parte, es fácil suponer, que si bien los asentamientos documentados no debieron ser contemporáneos durante la totalidad de sus ocupaciones, sí al menos

(18) No descartamos la localización de algún nuevo asentamiento cuando iniciemos los trabajos de prospección intensiva, si bien creemos que dicha circunstancia no afectaría de manera significativa a las conclusiones aquí expuestas. 
lo debieron ser en un momento de las mismas, como así además parecen corroborarlo las dataciones radiocarbónicas obtenidas hasta el momento en los yacimientos excavados (19), que indican su contemporaneidad en torno al 1.500 a.C. Y por último, las características de los yacimientos documentados, con una presencia importante de estructuras, permite estimar el tamaño de los mismos, si no al menos con valores absolutos, sí para realizar una evaluación fiable en términos comparativos. Además, en el caso de las Lagunas de Ruidera, a la mera diferenciación de tamaños, podemos unir otros aspectos relacionados con ésta como indicadores de jerarquización. Éste sería el caso de la existencia de una relación de proporcionalidad inversa entre tamaño y proximidad, así como de proporcionalidad directa entre tamaño y contactos visuales (20). Respecto al primero, cabe mencionar que los yacimientos localizados en el entorno de los más grandes, como Cerro Chicano y Mesa del Almendral, rara vez superan los $500 \mathrm{~m}^{2}$. Respecto al segundo, cabe indicar que los yacimientos de mayor tamaño presentan contacto visual con un mínimo de 2 yacimientos, mientras que el resto con uno, o ninguno.

Diferencias en el tamaño de los asentamientos están bien documentadas en La Mancha y zonas limítrofes durante la Edad del Bronce (Chapman, 1991; Díaz-Andreu, 1994; Fernández-Miranda et al., 1994; Ruiz Taboada, 1998) si bien éstas no siempre han sido interpretadas como un indicio de jerarquización. Así, en La Mancha Oriental (Fernández-Miranda et al., 1994), territorio limítrofe con el nuestro, las diferencias de tamaño son interpretadas como el resultado de la escisión de unos grupos respecto a otros mayores (Fernández-Miranda et al., 1994), mecanismo, por otra parte habitual, entre las comunidades con un modo doméstico de producción para solventar sus tensiones internas (Rovira y Santacana, 1980). En nuestro caso, creemos que las diferencias de tamaño no pueden ser del

(19) A este respecto cabe indicar que la totalidad de ellos son yacimientos de cierta envergadura, careciendo totalmente de información sobre yacimientos de reducidas dimensiones (inferiores a los $500 \mathrm{~m}^{2}$ ). Cabe pensar que éstos debieron sufrir una ocupación sensiblemente más reducida que los yacimientos excavados. A pesar de ello, seguimos considerando que éstos debieron estar ocupados en torno a mediados del II milenio a.C., que sin duda, debió representar el momento álgido en la ocupación de este territorio durante la Edad del Bronce.

(20) Recordamos la importancia de éstos, que motivan el emplazamiento de Altarejos-2 en una zona sin defensas naturales, en la que es posible el contacto visual con otros dos asentamientos, desestimando otras con mejores defensas -patrón normal-, pero en las que el contacto visual sólo era posible con uno. todo interpretadas en estos términos, ya que el alto índice de solapamiento documentado entre los territorios de explotación -afectan en la mayoría de los casos a las tierras más próximas a los asentamientos- no favorecería el fin último de la escisión, es decir, la eliminación de tensión, que en este caso se trasladaría a los vecinos más próximos.

Otro aspecto a destacar a favor de la existencia de jerarquización territorial es el lugar elegido para el emplazamiento de los asentamientos. La mayoría de éstos se localizan en lugares con un buen control visual sobre el fondo del valle en el que se emplazan. Dadas las características geológicas que presentan los valles en esta zona, creemos que esta circunstancia no puede ser interpretada en términos meramente subsistenciales, dadas las escasas posibilidades de aprovechamiento agrícola que presentan. Más bien parece tener un origen estratégico, que habría que buscar en la necesidad de ejercer un control sobre éstos como vías de comunicación.

Un tercer áspecto a favor de la existencia de jerarquización territorial sería la existencia de una posible diferenciación funcional entre los asentamientos. Ésta, desde nuestro punto de vista, sería la explicación para la localización de algunos asentamientos, como Huerta de Aguas-2 y Casa del Gavilán -sin control visual sobre el fondo del valle, pero rodeados de tierras óptimas para el cultivo-; también para los localizados en los fondos de valle, buscando ubicaciones en ambientes palustres que favorecerían el aprovechamiento de su entorno en verano, sin por otra parte dejar de ejercer un control sobre los fondos de valle; y por último para Cuesta de la Almagra, al localizarse sobre un cerrete de cuarcita -único afloramiento de la zona-, lo que nos hace pensar que podría estar relacionado con la explotación de la cuarcita como materia prima para la talla (21) e incluso con la posible explotación de las manchas de azurita y malaquita en el documentada (22).

Finalmente, el último factor a favor de la jerarquización territorial sería la existencia de una probable apropiación de excedentes por parte de los asentamientos de mayor tamaño, como Cerro Chicano y la Mesa del Almendral, que vendría indicada por la ausencia de proporcionalidad entre el tamaño de estos asentamientos y el de sus territorios de

(21) Ésta está presente en todos los yacimientos de la zona como principal soporte para la talla, superando al sílex y a la procedente de cantos de río.

(22) Información proporcionada por la Hoja Explicativa del Mapa Geológico Nacional n. 788. 
explotación. Esta apropiación estaría indicando la existencia de un control político de estos asentamientos sobre los de tamaño más reducido, control que también vendría avalado por la importancia que parecen adquirir los contactos visuales, que como ya vimos, lleva a condicionar la localización de algunos asentamientos, tal y como sucede en el caso de Altarejos-2. Este control político tendría su reflejo arqueológico en un territorio jerarquizado.

Por tanto, y a tenor de los aspectos anteriormente analizados, creemos plausible admitir la hipótesis de la existencia de una organización jerárquica en el territorio de las Lagunas de Ruidera durante la Edad del Bronce. Ahora bien, ¿cuáles pudieron ser las causas que provocaron dicha organización? Desde nuestro punto de vista, la concentración de yacimientos documentada en esta zona y su modelo de ocupación del territorio no parece estar relacionada con el potencial agrícola de estas tierras, muy escaso, por otra parte. Así parecen corroborarlo las pocas posibilidades de aprovechamiento que presentan los fondos de los valles - suelos con mayor potencial agrícola-, así como el hecho de que en la mayoría de los yacimientos que presentan tierras aptas para el cultivo en sus territorios de explotación, éstas se localicen en las zonas más alejadas. Si a ello unimos la predilección por la ubicación en zonas con un buen control visual sobre los fondos de los valles, parece más acertado relacionar el patrón documentado con la existencia de otros factores que harían atractivo este territorio, entre los cuales sin duda el principal podría ser su valor estratégico como zona de aprovisionamiento de pastos, especialmente durante el período estival. La importancia de estos territorios como zona de aprovisionamiento de pastos, así como de paso hacia otras zonas, se puede rastrear históricamente a partir del importante papel jugado por el castillo de Peñarroya dentro de las posesiones manchegas de la Orden de S. Juan. Éste se convirtió en el enclave más importante debido a los numerosos ingresos que generaba derivados, tanto de la explotación de las dehesas bajo su jurisdicción, como por el control sobre el paso de ganados que circulaban por el valle del Alto Guadiana camino principalmente de las Lagunas de Ruidera y la sierra de Alcaraz (Ruibal, 1993; Serrano, 2000).

La posible existencia de una trashumancia en tiempos prehistóricos hacia las Lagunas de Ruidera en busca de pastos de verano ya ha sido considerada por otros autores (Harrison y Moreno, 1985: 66). Éstos la sitúan dentro de un modelo, según el cual durante el II milenio a.C. en la Península Ibérica se generaliza un sistema económico basado en el intercambio de animales y sus productos. Dentro de éste, la explotación animal depende de los derechos que dan acceso a los pastos y prados, los cuales a su vez están sujetos a la demanda de éstos (Harrison y Moreno, 1985: 80). La incipiente jerarquización documentada en el territorio de las Lagunas de Ruidera parece encajar bien dentro de este modelo, sobre todo si tenemos en cuenta la alta densidad de población que debió existir en el territorio limítrofe de La Mancha durante el II milenio a.C. La misma puede deducirse del importante número de yacimientos conocidos, a los que habría que unir los ya desaparecidos como consecuencia de la actividad agrícola, tal y como recientemente hemos podido comprobar en el mismo valle del Alto Guadiana, unos kilómetros al norte de la zona estudiada (23). Parece aceptado que las comunidades que habitaban las llanuras manchegas practicaban una economía de tipo intensivo. En ésta, la ganadería debió jugar un papel importante, tal y como parecen poner de manifiesto los restos de fauna recuperados en las motillas del Azuer y Los Palacios, los cuales parecen mostrar la utilización del ganado no sólo para la provisión de carne, sino también para la provisión de los denominados productos secundarios, como la leche, la lana o la fuerza motriz (Harrison y Moreno, 1985).

Este modelo de intensificación en un medio como el de La Mancha, con un importante déficit hídrico -menos cantidad de agua precipitada que evaporada-(Juárez y Ponce, 1987) es muy probable que desencadenara desequilibrios entre la demanda de pastos y la cantidad disponible de éstos en los períodos estivales, momento en el que el déficit hídrico se hace más acusado. Estos desequilibrios convertirían a un territorio como el de las Lagunas de Ruidera en una zona muy atractiva para las comunidades manchegas, ya que aquí el déficit hídrico se ve compensado por la presencia de las lagunas. Ello, unido a su proximidad a los territorios manchegos de mayor densidad de población, hace factible pensar en el desarrollo de una trashumancia hacia éstas en los períodos estivales, así como su conversión en una importante vía de paso hacia otros territorios con pastos estivales como el Campo de Montiel o la sierra de Alcaraz. Estos movimientos de ganado provocarían un aumento de la

(23) Aquí, recientemente, hemos tenido noticia de tres nuevas motillas, de las que dos ya han sido desmanteladas por el laboreo agrícola. Ver notas 8 y 11 .

T. P., 59, n. ${ }^{\circ} 1,2002$ 
presión sobre los recursos existentes en las Lagunas de Ruidera, lo que llevaría a las comunidades que las habitaban a ejercer un control más estricto, tanto sobre sus recursos como sobre las vías de acceso y tránsito. Esta presión debió jugar un importante papel dinamizador en el proceso, tal vez ya iniciado, de desarrollo de una desigualdad social, que tendrá su principal reflejo arqueológico en la jerarquización del territorio.

Por todo lo expuesto con anterioridad, consideramos que el patrón de poblamiento observado en las Lagunas de Ruidera y sus posibles causas constituyen una buena hipótesis de partida que convierte a este territorio en una zona de gran interés para profundizar en el estudio y análisis de los sistemas económicos desarrollados por las poblaciones manchegas durante la Edad del Bronce, y sus implicaciones en el nivel social.

\section{BIBLIOGRAFÍA}

CAPEL, J. (1987): "Estudio mineralógico y geoquímico de sedimentos y cerámicas arqueológicas de algunos yacimientos de La Mancha". Oretum II 1986: 55-156.

CHAPMAN, R. (1991): La formación de las sociedades complejas. Crítica. Barcelona.

Colmenarejo, R.; Galán, C.; Martínez, J. y Sánchez, J. (1988): "La motilla de St ${ }^{a}$ María del Retamar (Argamasilla de Alba, Ciudad Real)". Oretum III 1987: 79-109.

DíAz-Andreu, M. (1994): La Edad del Bronce en Cuenca. Serie Arqueología Conquense 13. Diputación de Cuenca. Cuenca

Fernández-Miranda, M.; Fernández-Posse, M. ${ }^{a}$ D.; Gilman, A. y Martín, C. (1994): "La Edad del Bronce en la Mancha Oriental”. Actas del Simposium La Edad del Bronce en Castilla-La Mancha (Toledo 1990): 243287. Toledo.

Galán, C. y SánChez, J. (1994): "Santa María del Retamar 1984-1994”. En J. Sánchez., C. Galán, A. Caballero, C. Fernández y M. ${ }^{\mathrm{a}} \mathrm{T}$. Musat (coord.): Arqueología en Ciudad Real. Patrimonio Histórico-Arqueología, 8. Junta de Comunidades de Castilla-La Mancha. Toledo: $87-$ 110.

GonzÁlez, J.A.; Ordóñez, S. y García del Cura, Ma ${ }^{\mathrm{a}}$.A. (1987): "Evolución geomorfológica de las Lagunas de Ruidera (Albacete-Ciudad Real)". Estudios Geológicos 43: 281-284.

Gutiérrez, C.; Gómez, A.J. y Ocaña, A. (2000): “El enterramiento múltiple del Abrigo de Cueva Maturras (Argamasilla de Alba)". En E. Benítez de Lugo (coord.): El Patrimonio Arqueológico de Ciudad Real. Univer- sidad Nacional de Educación a Distancia. Valdepeñas: 43-65

Harrison, R. y Moreno, G. (1985): "El policultivo ganadero o la revolución de los productos secundarios". Trabajos de Prehistoria 44: 51-82.

Hernando, A. y Galán, C. (1989): “Armas metálicas de la motilla de $\mathrm{St}^{\mathrm{a}}$ María del Retamar (Argamasilla de Alba, Ciudad Real)". Espacio, Tiempo y Forma 5: 191-221.

JimÉNEZ, S. y CHAPARRo, A. (1989): Las Lagunas de Ruidera en el tiempo. Villanueva de los Infantes.

JuÁrez, C. y Ponce, G. (1987): "La aridez factor climático limitativo de la agricultura en Castilla-La Mancha". El espacio rural en Castilla-La Mancha, I: 83 y ss.

LóPEZ, F.J. y FERnánDEZ, M. (1994): "El poblamiento de las Lagunas de Ruidera durante la Edad del Bronce". Actas del I Symposium La Edad del Bronce en Castilla-La Mancha (Toledo 1990): 365-374. Toledo.

Montero, I.; Rodríguez, S. y RoJAs, J.M. (1990): Arqueometalurgia de la provincia de Toledo: minería y recursos minerales de cobre. Diputación Provincial de Toledo. Toledo.

NÁJERA, T. y Molina, F. (1977): "La Edad del Bronce en La Mancha. Excavaciones en las motillas del Azuer y Los Palacios campaña 1974". Cuadernos de Prehistoria de la Universidad de Granada 2: 251-302.

OcAÑA, A. y GómEZ, A.J. (2000): "Prospecciones en el Alto Guadiana (Lagunas de Ruidera, Albacete). Nuevas aportaciones al conocimiento del Bronce Final: el poblado de la Mesa del Almendral". $3^{\circ}$ Congreso de Arqueología Peninsular (Vila Real 1999). Actas V: $187-$ 201. Oporto.

Peinado, M. y Martínez, J.Ma . (1985): El paisaje vegetal de Castilla-La Mancha. Junta de Comunidades de Castilla-La Mancha. Toledo.

Rico, M.T.; López, F.J. y Serna, J.L. (1997): “Arqueología”. En Parque Natural de las Lagunas de Ruidera. Ecohábitat. Madrid: 245-278.

Rovira, J. y Santacana, J. (1980): “Reflexiones sobre 'economía' prehistórica aplicada a los grupos culturales del este peninsular: el modo doméstico de producción". Informació Arqueológica 33-34: 48-52.

Ruibal, A. (1993): "El castillo de Peñarroya. Un enclave hospitalario en La Mancha". Estudios de Historia y de Arqueología Medievales IX: 217-239.

Ruiz TABOADA, A. (1998): La Edad del Bronce en la provincia de Toledo: La Mancha y su entorno. Servicio Publicaciones Diputación de Toledo. Toledo.

Serrano, P. (2000): "Incidencia socioeconómica de los pastos de Argamasilla de Alba y Peñarroya en el Priorato de S. Juan" En A.Madrid y H.O'Donel (coord.): II Jornadas de la Orden de S. Juan (Arenas de S. Juan, 1998). Diputación Provincial de Ciudad Real. Ciudad Real: 65-88. 